Abstract

\title{
Tunneling as a Source for Quantum Chaos ${ }^{\dagger}$
}

\author{
Ofir Flom ${ }^{1}$, Asher Yahalom ${ }^{1,2}$, Haggai Zilberberg ${ }^{1}$, Lawrence Horwitz ${ }^{3}$ and Jacob Levitan ${ }^{1}$ \\ 1 Ariel University, Ariel, Israel \\ 2 Princeton University, Princeton, NJ, USA \\ 3 Tel Aviv University, Tel Aviv-Yafo, Israel \\ + Presented at the Entropy 2021: The Scientific Tool of the 21st Century, 5-7 May 2021; Available online: \\ https://sciforum.net/conference/Entropy2021/.
}

Published: 5 May 2021

Classical chaos is generally defined as exponential divergence of nearby trajectories causing instability in the orbits with respect to initial conditions. The wave function may be thought of as representing an ensemble of points in phase space and a fast spreading of the wave packet can be compared with a rapid or exponential separation of neighboring trajectories in the classical case. We use an one dimensional model of a square barrier embedded in an infinite potential well to demonstrate that tunneling leads to a complex behavior of the wave function and that the degree of complexity may be quantified by use of the spatial entropy function defined by $S=-$ Integral of $|\operatorname{Psi}(x, t)|$ squared $\ln \mid \operatorname{Psi}$ $(\mathrm{x}, \mathrm{t}) \mid$ squared $\mathrm{dx}$

Chaos is supposed to imply increase of the entropy and a rapid rise of the entropy function can be understood as the burst of chaotic behavior. There is no classical counterpart to tunneling but a decrease in the tunneling may be interpreted as an approach of a quantum system to a classical system.

We show that changing the square barrier with barriers of increasing height/breadth not only decrease the tunneling but also slows down the rapid rise of the entropy function which for a low/thin barrier for small times is a fluctuating function around a smooth almost constant asymptotic value.

Also the mean square width of the wave packet shows a rapid rise for a low/thin barrier before entering a steady asymptotic mean.

We conclude that the complex behavior associated with tunneling and the rapid rise of entropy is similar to that expected from a chaotic dynamical system. We therefore suggest that the rapid spread of the wave function can be used as a definition for quantum chaos.

(C) 2021 by the authors. Licensee MDPI, Basel, Switzerland. This article is an open access article distributed under the terms and conditions of the Creative Commons Attribution (CC BY)

license (http://creativecommons.org/licenses/by/4.0/). 